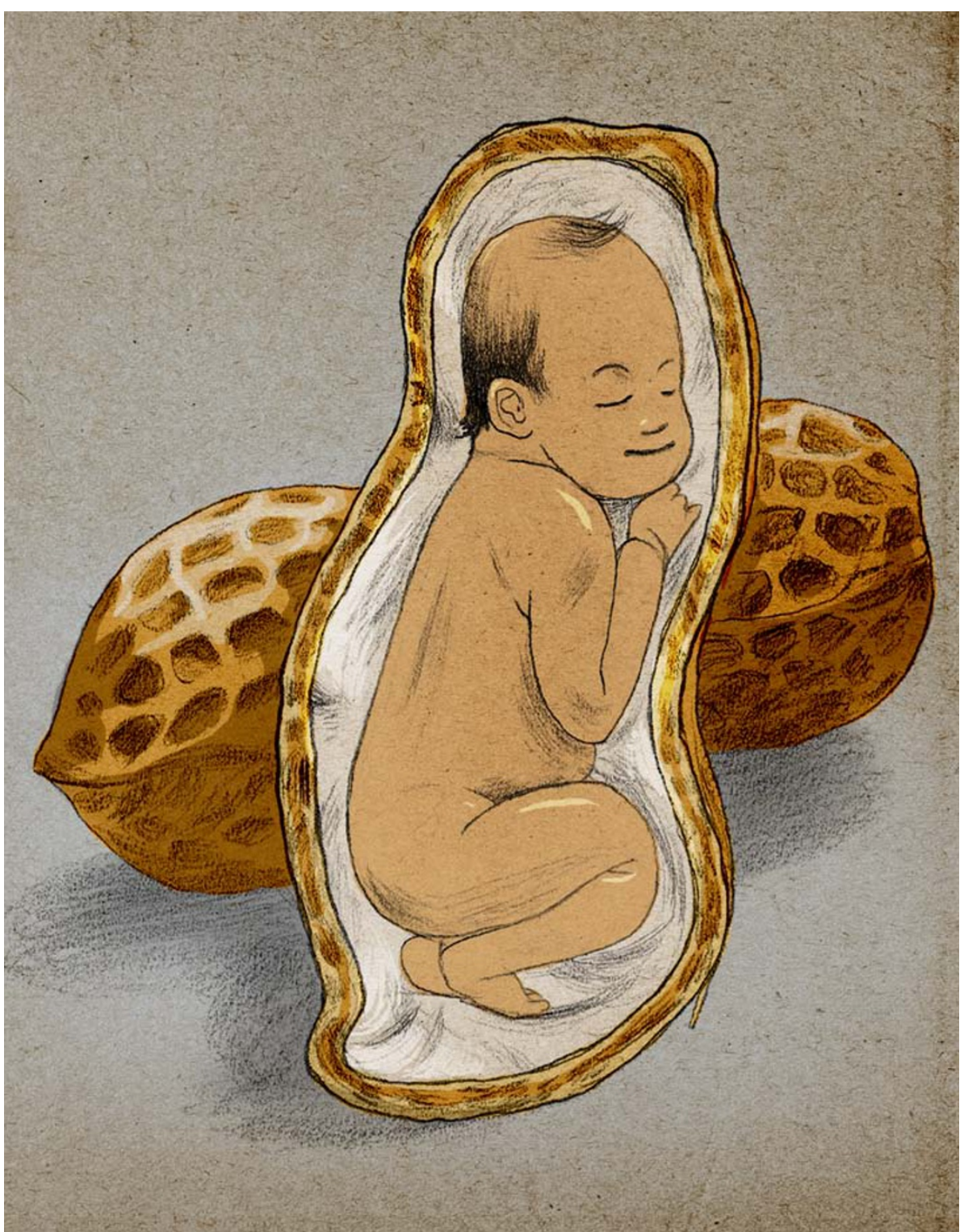

FOOD

\section{Picky eaters}

\section{Clinical trials are testing how careful exposure could protect people with potentially lethal allergies to everyday fare.}

\section{BY REBECCA KESSLER}

A spring roll may seem to be an innocent appetizer, but the shadow of death lurks between its crispy golden layers for the peanut allergy sufferer. The peanut butter sometimes used as spring roll sealant can send unwitting victims into the throes of anaphylaxis. At first, their skin may break out in hives. Their lips, tongue, and throat may start to tingle or itch. Then, in the worst cases, blood pressure drops, breathing becomes once only common in children younger than five or six years of age, now often endure into adolescence. The reasons remain obscure, but the costs are clear: in the United States, where food allergies are the leading cause of anaphylaxis, the cost of healthcare and lost productivity amounted to US\$510 million in 2007, according to estimates in a Virginia Commonwealth University study published in April 2011.

\section{ADVICE IN FLUX}

Babies with a known allergy-related disease, including eczema, or who have either a sibling or parent afflicted, are considered at high risk for developing food allergies. Until recently, it was standard practice in several developed countries to advise mothers of such children to avoid eating potential allergens during pregnancy and while breastfeeding; breastfeed infants exclusively for 4-6 months; and introduce potential allergens to a baby's diet only after $2-3$ years of age. The idea was to "wrap the infant up in a sort of immunological cocoon and not expose them to proteins that could launch allergic reactions," says Gideon Lack, professor of paediatric allergy at King's College London.

However, research suggests this could be the entirely wrong approach. "There is a possibility that we were achieving the reverse of our intentions through this avoidance policy," says Lack. A 2008 study that Lack co-authored showed that the incidence of peanut allergies among Jewish children in Israel is tenfold lower than among Jewish children in the United Kingdom, although young Israelis eat a great deal more food containing peanuts. This and other research led the American Academy of Pediatrics to revise its recommendations to its members in 2008 , to state that there is insufficient evidence to support restricting either the mother's diet during pregnancy and lactation or the infant's diet after 4-6 months. The UK Department of Health followed suit in 2009.

"Some mothers, when they have a peanutallergic child, say: 'I don't want to expose my [other] child to peanuts.' We can cite studies that suggest that may be the way to go. If they say 'no, I want to eat everything, we can cite studies that say that may be the way to go," says Hugh Sampson, director of Mount Sinai School of Medicine's Jaffe Food Allergy Institute in New York and a specialist in paediatric allergy. "The biggest thing is not to let mothers feel guilty about whatever choice they make, because at this point we really

Three large studies currently underway aim to resolve the lingering uncertainty about when, and whether, to expose children to potential allergens. Lack leads the Learning Early About Peanut Allergy (LEAP) study, which began in 2006 and is due to publish results in 2013. It follows 640 babies, half of whom are considered high risk for food allergies, to find out whether eating peanutcontaining foods during infancy leads to peanut
D NATURE.COM For some of the latest research on allergies to food: go.nature.com/2qzyoz don't know the best answer." 
allergies. Lack is also leading the Enquiring About Tolerance (EAT) study, due to report in 2015, probing the best time for infants to begin eating solid foods in general. EAT is comparing two strategies to see whether exclusive breastfeeding up to 6 months of age better prevents allergies than breastfeeding but also introducing 6 potentially allergenic foods at 3 months of age.

A third study is tracking more than 500 babies who tested positive for allergies to milk or egg, which puts them at high risk for developing peanut allergy. It aims to illuminate the role of maternal diet by seeing which babies go on to develop peanut allergies. Interim results published in 2010 seem to support the "cocoon hypothesis" that it is best to minimize exposure to allergens, since infants whose mothers ate more peanuts during pregnancy had higher levels of the peanut antibody in their blood, but it remains to be seen whether more of them will develop full-blown peanut allergies, says Sampson, a principal investigator of the study.

\section{REDUCING SENSITIVITY, CHASING TOLERANCE}

Attempts to treat allergy sufferers by exposing them to incremental doses of the substance that torments them date back a century. Allergy shots, which physicians administer to treat allergies to dust, pollen, pet dander and other irritants, are a prime example of the technique, called desensitization or immunotherapy. But researchers have trodden warily in applying it to food allergies, because the reactions can be so severe.

The death by anaphylaxis of a 15-yearold volunteer in a study on peanut injection immunotherapy in 1991 spread a pall over the field. John Oppenheimer, an allergist at the University of Medicine and Dentistry of New Jersey, led that ill-fated study. Twenty years later, he is sad that patients are still languishing in a "timebomb-like existence" where a mouthful of the wrong food could mean death, though he is heartened by recent research progress. "I tell this to all my young patients," Oppenheimer says. "I'm hopeful that by the time they go to college I'll have something to offer them that's better than avoidance, which is all I have right now."

If injecting food allergens is too risky for therapy, what about administering a tiny controlled dose with food? This seemingly more obvious avenue of delivery has yielded some positive results. In March 2011, researchers at the University of Cambridge, United Kingdom, reported that an oral immunotherapy trial of 22 peanut-allergic children fed incremental doses of peanut flour each day over the course of 30 weeks had raised their median tolerance by a factor of 1,000 - enough to abate trouble after any accidental ingestion. After treatment, 14 of the 22 children had no allergic reaction to eating the equivalent of about 32 peanuts, and another 4 children had only mild or moderate reactions. Also in March 2011, researchers at Duke University Medical Center in Durham,

\section{PREVALENCE OF FOOD ALLERGIES AMONG CHILDREN}

\begin{tabular}{|ll|l|l|}
\hline Reporting one or more food allergy (\%) & & USA & France \\
\hline Reporting specific allergy to (\%): & Peanut & 8.00 & 6.70 \\
& Milk & 2.02 & 0.55 \\
& Shellfish & 1.69 & 0.80 \\
\hline
\end{tabular}

North Carolina, reported early results from an ongoing oral immunotherapy study involving 25 peanut-allergic children. After a year, the 16 children in the test group all ate the equivalent of 20 peanuts, with only one suffering a mild reaction, whereas five of the nine taking placebos could still tolerate little or none, and the median tolerance of the placebo group was just one peanut.

In May 2011, Sampson's group reported the results of a study on a similar approach sometimes referred to as 'natural immunotherapy'. Over a 5-year period, 70 children allergic to milk were fed muffins and waffles, and later cheese pizzas, containing milk baked at progressively lower temperatures. Children in the treated group were 16-times more likely to tolerate uncooked milk than those in the control group.

Oral immunotherapy seems to help about $80 \%$

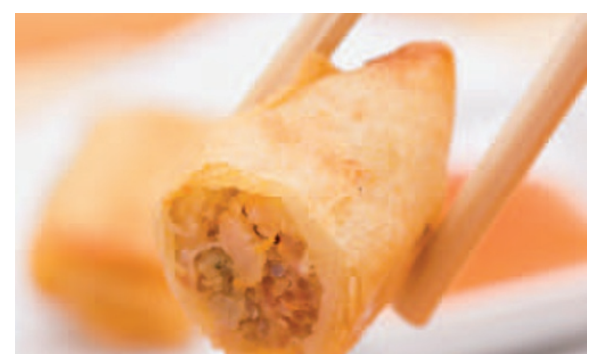

Spring rolls: not always a treat for the allergic.

of people tested so far, according to Sampson. Adjusting the treatment regime could help the remainder. In clinical trials, Sampson, Lack and others have shown that the safety and efficacy of this therapy improve when subjects take the drug omalizumab, a treatment developed for allergic asthma. Widespread use of omalizumab for this purpose, however, would be hugely expensive; its average wholesale cost in the United States works out at roughly US $\$ 20,000$ per patient per year. Researchers are also investigating the delivery of allergens in liquid form under the tongue or through a skin patch. Both approaches seem to reduce adverse reactions, Sampson says.

However, despite encouraging results, immunotherapy studies have so far tended to be small, lack sufficient controls, and be of too limited duration to reveal any long-term effects of treatment. There's also little evidence of any improved tolerance persisting if the regular allergen dose is discontinued; typically the effects wear off after anything from 1 week to
2 months, according to Sampson. "That's what people don't really understand," says Wesley Burks, director of the Duke University group. "They see the desensitization effect and they think it's a more permanent cure."

\section{INTOTHE CLINIC}

In an effort to hasten the arrival of a safe treatment for food allergies, the US not-for-profit group Food Allergy Initiative (FAI), based in New York, gathered about 40 allergists, policy makers, allergy advocates and regulators from the US Food and Drug Administration (FDA) to debate the best way forward. The group's consensus, according to FAI's executive director Mary Jane Marchisotto, was that out of several experimental treatments - including a Chinese herbal formula, a parasitic worm, and vaccines - oral immunotherapy has the best prospects. Subsequently, FAI has undertaken a campaign to orchestrate and raise funding for a phase III clinical trial in hundreds of subjects to lay the scientific groundwork for approval of an oral immunotherapy regimen by the FDA.

Sampson, who participated in the FAI gathering and receives FAI funding, estimates that this trial might yield an approved treatment within seven years. He notes, however, that the Chinese herbal formula developed, patented, and tested by his team, has promise and might bear fruit sooner. His colleagues are currently enrolling subjects to test it against several food allergies. If the herbal formula passes trials, it could be brought to market as early as 2013. As a herbal supplement, unlike a drug, it doesn't need to go through the lengthy and stringent FDA approval process, although the team does intend to pursue FDA approval in the long run.

Meanwhile, Burks says more than 100 families are lined up to enrol in his oral immunotherapy studies. At this stage, however, he is concerned when he hears of allergists using oral immunotherapy on patients outside the watchful supervision of controlled trials at major medical centres, and even of desperate parents attempting to treat their food-allergic kids at home. This is a bad idea, he warns, since serious adverse reactions can occur. "The use of oral immunotherapy should be still investigational," Burks says. "It's really not ready for practice."

Rebecca Kessler is a freelance journalist in Providence, Rhode Island. 